\title{
Enantiodivergent Pd-catalyzed C-C bond formation enabled through ligand parameterization
}

\author{
Shibin Zhao, ${ }^{1,2 *}$ Tobias Gensch, ${ }^{3 *}$ Benjamin Murray, ${ }^{1,2}$ Zachary L. Niemeyer, ${ }^{3}$ Matthew S. Sigman, ${ }^{34}$ \\ Mark R. Biscoe ${ }^{1,2 \dagger}$ \\ 1Department of Chemistry, The City College of New York, 160 Convent Avenue, New York, NY 10031, USA. ²Ph.D. Program in Chemistry, The Graduate Center of the City \\ University of New York, 365 Fifth Avenue, New York, NY 10016, USA. ${ }^{3}$ Department of Chemistry, University of Utah, 315 South 1400 East, Salt Lake City, UT 84112 , USA. \\ *These authors contributed equally to this work. †Corresponding author. Email: sigman@chem.utah.edu (M.S.S.); mbiscoe@ccny.cuny.edu (M.R.B.)
}

Despite the enormous potential for the use of stereospecific cross-coupling reactions to rationally manipulate the three-dimensional structure of organic molecules, the factors that control the transfer of stereochemistry in these reactions remain poorly understood. Herein we report a mechanistic and synthetic investigation into the use of enantioenriched alkylboron nucleophiles in stereospecific Pdcatalyzed Suzuki cross-coupling reactions. By developing a suite of molecular descriptors of phosphine ligands, we could apply predictive statistical models to select or design distinct ligands that respectively promoted stereoinvertive and stereoretentive cross-coupling reactions. Stereodefined branched structures were thereby accessed through the predictable manipulation of absolute stereochemistry, and a general model for the mechanism of alkylboron transmetallation was proposed.

Palladium-catalyzed cross-coupling reactions have revolutionized the construction of $\mathrm{C}\left(s p^{2}\right)-\mathrm{C}\left(s p^{2}\right)$ bonds. Among these cross-coupling processes, the Suzuki-Miyaura reaction has found particularly broad application due to its extensive reaction scope, as well as the stability, availability, and low toxicity of organoboron reagents (1). The 2010 Nobel Prize in chemistry was awarded, in part, to recognize the transformative impact of the Suzuki cross-coupling reaction on chemical synthesis. However, although $\mathrm{C}\left(s p^{2}\right)-\mathrm{C}\left(s p^{2}\right)$ bond construction is now considered routine using the Suzuki reaction, extension of this process to the formation of $\mathrm{C}\left(s p^{3}\right)-\mathrm{C}\left(s p^{2}\right)$ bonds using alkylboron nucleophiles remains a significant challenge. Of particular interest, a variant using secondary alkylboron nucleophiles with predictable and controllable stereospecificity would establish a powerful synthetic strategy to access molecular geometries with precise three-dimensional control, expanding the exceptional capabilities of the Suzuki reaction (Fig. 1A).

Many efforts have focused on the use of enantioenriched secondary alkylboron nucleophiles in Suzuki cross-coupling reactions (2-4). Significant limitations remain due to slow transmetallation of the highly covalent and sterically congested $\mathrm{C}\left(s p^{3}\right)$-B bond in these reagents, as well as the propensity of the resulting Pd-alkyl species to undergo $\beta$-hydride elimination/reinsertion sequences, which can result in isomerization of the alkyl group and racemization of the stereocenter. To circumvent prohibitively slow transmetallation, as well as competing $\beta$-hydride elimination/reinsertion pathways, most stereospecific Suzuki reactions have required the use of secondary alkylboron nucleophiles that are electronically activated via inclusion of a $\mathrm{C}\left(s p^{2}\right) \alpha$-carbon, an $\alpha$-heteroatom, and/or a strongly coordinating $\beta$-carbonyl group (5-17). In addition, alkylboron nucleophiles can undergo transmetallation via either stereoretentive or stereoinvertive pathways depending on the nature of the substrate, catalyst, and/or reaction conditions. In many cases, the factors controlling the dominant mechanism of transmetallation are not understood (Fig. 1B). Thus, a predictive stereochemical model for transmetallation of alkylboron reagents remains elusive.

Recently, we reported a stereospecific Pd-catalyzed crosscoupling reaction using unactivated secondary alkylboron nucleophiles (18). With $\mathrm{P}^{t} \mathrm{Bu}_{3}$ as a supporting ligand, enantioenriched arylation products were obtained with transmetallation proceeding primarily via a stereoinvertive mechanism. Whereas several enlightening mechanistic studies have recently been conducted on the transmetallation of arylboron nucleophiles (19-23), these studies have not addressed the transmetallation of alkylboron nucleophiles in $\mathrm{C}\left(s p^{3}\right)-\mathrm{C}\left(s p^{2}\right)$ bond-forming processes $(24,25)$. Thus, unactivated alkylboron nucleophiles constitute an attractive starting point from which to investigate the reaction parameters most influential to the mechanism of alkylboron transmetallation. This mechanistic work should simultaneously facilitate the development of new synthetic methods to rationally incorporate/manipulate stereocenters via cross-coupling strategies. To this end, we report a study using predictive statistical models $(26,27)$ to relate phosphine ligand properties to stereochemical outcomes obtained from Pd-catalyzed Suzuki reactions of unactivated enantioenriched secondary alkylboron 
nucleophiles and aryl electrophiles. With statistical models that rely on a next generation set of molecular descriptors, we achieved a stereoretentive Pd-catalyzed cross-coupling reaction of such nucleophiles. Furthermore, we have identified an improved ligand for the stereoinvertive variant, thus enabling an entirely ligand-controlled enantiodivergent process from a single-enantiomer organoboron nucleophile (28). Our statistical models also provide compelling evidence that each transmetallation pathway is intimately tied to specific electronic properties of the supporting ligand, which serves as a predictive guide to the mechanism of alkylboron transmetallation to palladium.

Initial investigations using electronically differentiated aryl chlorides with enantioenriched ${ }^{s} \mathrm{BuBF}_{3} \mathrm{~K}$ revealed a trend correlating diminished stereofidelity with the use of more electron-deficient coupling partners (Fig. 1C, I). This observation suggested that subtle electronic effects could influence the mechanism of transmetallation and the resulting stereochemical outcome. Additionally, when the phosphine ligand was varied in an initial screen with a common aryl chloride electrophile, a considerable change in the reaction outcome from stereoinvertion to stereoretention was found (Fig. 1C, II). No obvious correlation was observed between these results and the steric properties (solid angle) of the ligand. Taken together, these outcomes were difficult to interpret and inspired the use of ligand parameterization tools to provide a platform for both predictive ligand performance and mechanistic interrogation.

An expanded inventory of common phosphines with varied properties was evaluated in the Suzuki reaction of enantioenriched ${ }^{s} \mathrm{BuBF}_{3} \mathrm{~K}$ and ethyl 4-chlorobenzoate. This dataset was then subjected to correlation analysis of phosphine structural features with the stereochemical outcomes as well as the ratios of branched:linear products in these reactions. We devised a workflow and universal parameter set to describe the catalyst properties from the phosphine itself (29-32). The workflow was initiated by performing a molecular mechanics (MM) conformational search to reveal representative low energy conformers. (Fig. 2A). Next, geometry optimization of the conformers using DFT was followed by parameter collection. Subsequently, four descriptor subsets were defined to capture the conformational dynamics of the ligands by including the mathematical extreme descriptor values (minimum and maximum), the lowest energy conformer values, and the Boltzmann weighted averages. We viewed the unique treatment of representative conformers as a crucial means of describing ensemble properties such as chemical shift while also probing structural flexibility during catalysis.

The final step in the workflow involved the analysis of both the stereofidelity and the branched:linear product ratio. These two readouts presumably describe two stages of the reaction mechanism (Fig. 1B): i) the competing stereoretentive and stereoinvertive transmetallation mechanisms that determine the final stereochemistry of the cross-coupling product and ii) the competitive $\beta$-hydride elimination/isomerization sequences that follow transmetallation. A correlation of the branched:linear ratio with the final enantiopurity of the product reveals that $\beta$-hydride elimination is responsible for both racemization and isomerization to the linear side product. Furthermore, a modest trend is observed relating the minimum width $B_{1}$ of the phosphine ligand to the branched:linear ratio (Fig. 2B). This is consistent with reports of large ligands facilitating reductive elimination over $\beta$-hydride elimination (33), and suggests the use of a parameterization approach to take into account the conformational flexibility of ligands.

Since the inherent selectivity of the transmetallation mechanism is masked by deleterious racemization as a consequence of $\beta$-hydride elimination, only ligands providing high selectivity were further investigated ( $>30 \%$ ee, Fig. $2 \mathrm{~B}$ ). The molecular electrostatic potential minimum in the phosphorus lone pair region $\left(V_{\min }\right)$ has been shown to correlate with the classical Tolman electronic parameter (34). Thus, $V_{\min }$ serves as an easily computable measure for the overall ligand electronics. A correlation between enantioselectivity and $V_{\min }$ was observed within the abridged dataset indicating that electronic properties of the ligand determine the mechanism of transmetallation. Specifically, electron-rich trialkylphosphines promoted stereoinvertive reactions, whereas the electron-poorer triarylphosphines provided modest selectivity for stereoretention. Use of the bulky, electron-rich ligand $\operatorname{PAd}_{3}(\mathbf{9})$, which was recently reported by Carrow (35), resulted in a particularly large preference for the stereoinvertive outcome. Based on these data, we hypothesized and virtually evaluated ligands for improved stereoretentive outcomes with the following features: i) large ligand bulk to prevent $\beta$-hydride elimination and racemization, and ii) electron-deficient aryl substituents at phosphorus to promote the stereoretentive mechanism and to accelerate reductive elimination (Fig. 2B). Among the proposed ligands was a set of biaryl phosphines (11-15), as pioneered by Buchwald (36), featuring various electron-deficient aryl groups at phosphorus. Gratifyingly, ligands $\mathbf{1 1}$ and 14 promote the alkyl Suzuki cross-coupling reaction with significantly enhanced selectivity (up to $90 \%$ ee) and minimal alkyl isomerization. Thus, parameterization-driven optimization facilitated development of a stereoretentive Suzuki reaction involving unactivated alkylboron nucleophiles. When considered alongside the introduction of $\mathbf{9}$ to achieve stereoinvertive couplings, predictable control of the absolute sense of enantioselectivity (retention or inversion) can be engendered by simply selecting the appropriate ligand.

Our stereochemical investigations of secondary alkylboron transmetallation in the Suzuki reaction suggested that both enantiomers of a cross-coupling product could be 
selectively accessed through use of a single enantioenriched alkylboron reagent with the proper selection of the phosphine ligand. The scope of this process is depicted in Fig. 3. Using enantioenriched, unactivated alkyltrifluoroborate nucleophiles, ligand-controlled stereoselectivity was broadly achieved in cross-coupling reactions with aryl electrophiles. Strongly $\pi$-accepting ligands bis- $\mathrm{CF}_{3} \mathrm{PhSPhos}(\mathbf{1 1})$ and bis$\mathrm{CF}_{3} \mathrm{PhXPhos}$ (14), which emerged from our parameterization-guided optimization, preferentially promote the stereoretentive pathway, while strongly $\sigma$-donating ligand $\mathrm{PAd}_{3}$ (9) preferentially promotes the stereoinvertive pathway. Because electron-poor palladium catalysts commonly undergo slow oxidative addition with aryl chlorides, we also evaluated aryl bromide and triflate electrophiles in reactions involving $\mathbf{1 1}$ and 14. A particular highlight of this protocol is the uniformity of the conditions used for both the stereoinvertive and stereoretentive reactions: each operates in a toluene/water mixture as solvent, with a carbonate base, and no additional additives. Both reaction variants tolerated the use of electron-rich and electron-deficient aryl electrophiles, as well as an aryl electrophile bearing an ortho-substituent. High stereofidelity was achieved for all of these reactions, including those involving alkylboron nucleophiles bearing thiophenyl and phenoxide substituents. Use of an alkylboron nucleophile containing a larger substituent (replacing methyl with ethyl) at the stereogenic center was also well tolerated (16i). In the absence of a methyl substituent, the stereoinvertive variant shows modestly reduced selectivity that may be improved by reducing the reaction temperature. This was partially anticipated due to the greater sensitivity of the metal fragment to steric congestion at carbon in the stereoinvertive $\mathrm{S}_{\mathrm{E}} 2$ transmetallation mechanism. Diastereomeric products $\mathbf{1 7 a}$ and $\mathbf{1 7 b}$ could be generated from a single alkylboron diastereomer (37) using 14 and $\mathrm{PAd}_{3}$, respectively (Fig. 3B). In these reactions, replacement of ligand $\mathbf{1 4}$ with $\mathrm{PAd}_{3}$ resulted in a change in diastereoselectivity from 30:1 to 1:5, a $3.6 \mathrm{kcal} / \mathrm{mol}$ free energy of activation difference dependent only on the ligand identity. No erosion of specificity was observed for electron-deficient aryl substrates in stereoinvertive Suzuki reactions using $\mathrm{PAd}_{3}$, in contrast to analogous reactions using $\mathrm{P}^{t} \mathrm{Bu}_{3}$. Furyl and thiophenyl electrophiles are also compatible with our system (Fig. 3C). As an additional mechanistic probe, trans-2-methylcyclopentyltrifluoroborate was subjected to the stereodivergent reaction conditions. Because trans-2-methylcyclopentyltrifluoroborate is sterically impeded from undergoing stereoinvertive transmetallation, only the stereoretentive process using $\mathbf{1 4}$ should be mechanistically viable. Indeed, we observed that use of ligand $\mathbf{1 4}$ smoothly generates $\mathbf{1 8}$ with stereoretention, while use of $\mathrm{PAd}_{3}$ results in low alkylboron conversion.

To further probe the origin of the ligand-dependent enantiodivergent process, we interrogated the mechanism of transmetallation using the parameterization strategy described above (Fig. 4). To accomplish this, phenyl-substituted substrate $\mathbf{2 0}$ was selected due to enhanced performance and thus a greater output range. A singular aryl chloride electrophile was chosen for this analysis to avoid potential attenuation of the ligand effects by the influence of different counterions (e.g., bromide or triflate). Additionally, 24 ligands were tested, excluding smaller ligands to reduce the complexity associated with $\beta$-hydride elimination. Multivariate linear regression revealed that most of the outputs can be expressed in two readily interpretable terms that discriminate the transmetallation pathways: the average energy of the $\mathrm{P}-\mathrm{C}$ antibonding orbitals $E_{\sigma^{\star}(\mathrm{P}-\mathrm{C})}$, representative of $\pi$-back bonding, and the energy of the lone pair orbital of phosphorus $E_{\mathrm{LP}(\mathrm{P})}$, a measure of the ligand's $\sigma$-donation capability (Fig. 4B). This outcome suggests that the stereoinvertive pathway is dependent on strong $\sigma$-donation from the ligand, which may stabilize a two-coordinate, cationic palladium complex. Conversely, the stereoretentive pathway is enhanced by $\pi$-back bonding, which may stabilize the coordination of a $\pi$-donor ligand $\mathrm{X}$ (presumably $\mathrm{OH}^{-}$) to $\mathrm{Pd}$. Including two steric descriptors such as $B_{1}$ and $L$ improves the model fit by treating the competitive $\beta$-hydride elimination that occurs using smaller ligands and decreases the observed specificity. This becomes evident when the four smallest ligands in this dataset are removed from the analysis, which results in an excellent correlation using just the two electronic descriptors with the experimentally observed stereochemical outcomes (Fig. 4D). Multivariate regression analysis thereby provides compelling evidence for the electronic factors favoring each transmetallation mechanism and thus a guideline for future developments in stereospecific cross-coupling reactions.

\section{REFERENCES AND NOTES}

1. J. C. H. Lee, D. G. Hall, "State-of-the-art in metal-catalyzed cross-coupling reactions of organoboron compounds with organic electrophiles," in Metal-Catalyzed CrossCoupling Reactions and More, A. de Meijere, S. Bräse, M. Oestreich, Eds. (WileyVCH, ed. 3, 2014), vol. 1, pp. 65-132.

2. C.-Y. Wang, J. Derosaa, M. R. Biscoe, Configurationally stable, enantioenriched organometallic nucleophiles in stereospecific Pd-catalyzed cross-coupling reactions: An alternative approach to asymmetric synthesis. Chem. Sci. 6, 51055113 (2015). doi:10.1039/C5SC01710F Medline

3. C. Sandford, V. K. Aggarwal, Stereospecific functionalizations and transformations of secondary and tertiary boronic esters. Chem. Commun. (Camb.) 53, 54815494 (2017). doi:10.1039/C7CC01254C Medline

4. J. P. G. Rygus, C. M. Crudden, Enantiospecific and iterative Suzuki-Miyaura crosscouplings. J. Am. Chem. Soc. 139, 18124-18137 (2017). doi:10.1021/jacs.7b08326 Medline

5. D. Imao, B. W. Glasspoole, V. S. Laberge, C. M. Crudden, Cross coupling reactions of chiral secondary organoboronic esters with retention of configuration. J. Am. Chem. Soc. 131, 5024-5025 (2009). doi:10.1021/ja8094075 Medline

6. D. L. Sandrock, L. Jean-Gérard, C. Y. Chen, S. D. Dreher, G. A. Molander, Stereospecific cross-coupling of secondary alkyl $\beta$-trifluoroboratoamides. J. Am. Chem. Soc. 132, 17108-17110 (2010). doi:10.1021/ja108949w Medline

7. T. Ohmura, T. Awano, M. Suginome, Stereospecific Suzuki-Miyaura coupling of chiral $\alpha$-(acylamino)benzylboronic esters with inversion of configuration. J. Am. Chem. Soc. 132, 13191-13193 (2010). doi:10.1021/ja106632j Medline 
8. T. Awano, T. Ohmura, M. Suginome, Inversion or retention? Effects of acidic additives on the stereochemical course in enantiospecific Suzuki-Miyaura coupling of $\alpha$-(acetylamino)benzylboronic esters. J. Am. Chem. Soc. 133, 2073820741 (2011). doi:10.1021/ja210025q Medline

9. J. C. H. Lee, R. McDonald, D. G. Hall, Enantioselective preparation and chemoselective cross-coupling of 1,1-diboron compounds. Nat. Chem. 3, 894899 (2011). doi:10.1038/nchem.1150 Medline

10. B. M. Partridge, L. Chausset-Boissarie, M. Burns, A. P. Pulis, V. K. Aggarwal, Enantioselective synthesis and cross-coupling of tertiary propargylic boronic esters using lithiation-borylation of propargylic carbamates. Angew. Chem. Int. Ed. 51,11795-11799 (2012). doi:10.1002/anie.201203198 Medline

11. G. A. Molander, S. R. Wisniewski, Stereospecific cross-coupling of secondary organotrifluoroborates: Potassium 1-(benzyloxy)alkyltrifluoroborates. J. Am. Chem. Soc. 134, 16856-16868 (2012). doi:10.1021/ja307861n Medline

12. S. C. Matthew, B. W. Glasspoole, P. Eisenberger, C. M. Crudden, Synthesis of enantiomerically enriched triarylmethanes by enantiospecific Suzuki-Miyaura cross-coupling reactions. J. Am. Chem. Soc. 136, 5828-5831 (2014). doi:10.1021/ia412159g Medline

13. C. Sun, B. Potter, J. P. Morken, A catalytic enantiotopic-group-selective Suzuki reaction for the construction of chiral organoboronates. J. Am. Chem. Soc. 136 , 6534-6537 (2014). doi:10.1021/ja500029w Medline

14. T. P. Blaisdell, J. P. Morken, Hydroxyl-directed cross-coupling: A scalable synthesis of debromohamigeran $\mathrm{E}$ and other targets of interest. J. Am. Chem. Soc. 137 8712-8715 (2015). doi:10.1021/jacs.5b05477 Medline

15. S. Miyamura, M. Araki, T. Suzuki, J. Yamaguchi, K. Itami, Stereodivergent synthesis of arylcyclopropylamines by sequential $\mathrm{C}-\mathrm{H}$ borylation and Suzuki-Miyaura coupling. Angew. Chem. Int. Ed. 54, 846-851 (2015). doi:10.1002/anie.201409186 Medline

16. Y. Lou, P. Cao, T. Jia, Y. Zhang, M. Wang, J. Liao, Copper-catalyzed enantioselective 1,6-boration of para-quinone methides and efficient transformation of gemdiarylmethine boronates to triarylmethanes. Angew. Chem. Int. Ed. 127, 1230212306 (2015). doi:10.1002/ange.201505926

17. G. L. Hoang, J. M. Takacs, Enantioselective $\gamma$-borylation of unsaturated amides and stereoretentive Suzuki-Miyaura cross-coupling. Chem. Sci. 8, 4511-4516 (2017). doi:10.1039/C7SC01093A Medline

18. L. Li, S. Zhao, A. Joshi-Pangu, M. Diane, M. R. Biscoe, Stereospecific Pd-catalyzed cross-coupling reactions of secondary alkylboron nucleophiles and aryl chlorides. J. Am. Chem. Soc. 136, 14027-14030 (2014). doi:10.1021/ja508815w Medline

19. C. Amatore, A. Jutand, G. Le Duc, Kinetic data for the transmetalation/reductive elimination in palladium-catalyzed Suzuki-Miyaura reactions: Unexpected triple role of hydroxide ions used as base. Chemistry 17, 2492-2503 (2011). doi:10.1002/chem.201001911 Medline

20. B. P. Carrow, J. F. Hartwig, Distinguishing between pathways for transmetalation in Suzuki-Miyaura reactions. J. Am. Chem. Soc. 133, 2116-2119 (2011). doi:10.1021/ja1108326 Medline

21. A. A. Thomas, S. E. Denmark, Pre-transmetalation intermediates in the SuzukiMiyaura reaction revealed: The missing link. Science 352, 329-332 (2016). doi:10.1126/science.aad6981 Medline

22. A. A. Thomas, H. Wang, A. F. Zahrt, S. E. Denmark, Structural, kinetic, and computational characterization of the elusive arylpalladium(II)boronate complexes in the Suzuki-Miyaura reaction. J. Am. Chem. Soc. 139, 3805-3821 (2017). doi:10.1021/jacs.6b13384 Medline

23. A. A. Thomas, A. F. Zahrt, C. P. Delaney, S. E. Denmark, Elucidating the role of the boronic esters in the Suzuki-Miyaura reaction: Structural, kinetic, and computational investigations. J. Am. Chem. Soc. 140, 4401-4416 (2018). doi:10.1021/jacs.8b00400 Medline

24. B. H. Ridgway, K. A. Woerpel, Transmetalation of alkylboranes to palladium in the Suzuki coupling reaction proceeds with retention of stereochemistry. J. Org. Chem. 63, 458-460 (1998). doi:10.1021/j0970803d Medline

25. K. Matos, J. A. Soderquist, Alkylboranes in the Suzuki-Miyaura coupling: Stereochemical and mechanistic studies. J. Org. Chem. 63, 461-470 (1998). doi:10.1021/j0971681s Medline

26. C. B. Santiago, J.-Y. Guo, M. S. Sigman, Predictive and mechanistic multivariate linear regression models for reaction development. Chem. Sci. 9, 2398-2412 (2018). doi:10.1039/C7SC04679K Medline
27. M. S. Sigman, K. C. Harper, E. N. Bess, A. Milo, The development of multidimensional analysis tools for asymmetric catalysis and beyond. Acc. Chem. Res. 49, 1292-1301 (2016). doi:10.1021/acs.accounts.6b00194 Medline

28. S. Krautwald, E. M. Carreira, Stereodivergence in asymmetric catalysis. J. Am. Chem. Soc. 139, 5627-5639 (2017). doi:10.1021/jacs.6b13340 Medline

29. C. A. Tolman, Steric effects of phosphorus ligands in organometallic chemistry and homogeneous catalysis. Chem. Rev. 77, 313-348 (1977). doi:10.1021/cr60307a002

30. J. Jover, N. Fey, J. N. Harvey, G. C. Lloyd-Jones, A. G. Orpen, G. J. J. Owen-Smith, P. Murray, D. R. J. Hose, R. Osborne, M. Purdie, Expansion of the ligand knowledge base for monodentate P-donor ligands (LKB-P). Organometallics 29, 6245-6258 (2010). doi:10.1021/om100648v

31. Z. L. Niemeyer, A. Milo, D. P. Hickey, M. S. Sigman, Parameterization of phosphine ligands reveals mechanistic pathways and predicts reaction outcomes. Nat. Chem. 8, 610-617 (2016). doi:10.1038/nchem.2501 Medline

32. K. Wu, A. G. Doyle, Parameterization of phosphine ligands demonstrates enhancement of nickel catalysis via remote steric effects. Nat. Chem. 9, 779-784 (2017). doi:10.1038/nchem.2741 Medline

33. R. Jana, T. P. Pathak, M. S. Sigman, Advances in transition metal (Pd, Ni, $\mathrm{Fe})$ catalyzed cross-coupling reactions using alkyl-organometallics as reaction partners. Chem. Rev. 111, 1417-1492 (2011). doi:10.1021/cr100327p Medline

34. C. H. Suresh, N. Koga, Quantifying the electronic effect of substituted phosphine ligands via molecular electrostatic potential. Inorg. Chem. 41, 1573-1578 (2002) doi:10.1021/ic0109400 Medline

35. L. Chen, P. Ren, B. P. Carrow, Tri(1-adamantyl)phosphine: Expanding the boundary of electron-releasing character available to organophosphorus compounds. J. Am. Chem. Soc. 138, 6392-6395 (2016). doi:10.1021/jacs.6b03215 Medline

36. D. S. Surry, S. L. Buchwald, Dialkylbiaryl phosphines in Pd-catalyzed amination: A user's guide. Chem. Sci. 2, 27-50 (2011). doi:10.1039/C0SC00331J Medline

37. S. Balieu, G. E. Hallett, M. Burns, T. Bootwicha, J. Studley, V. K. Aggarwal, Toward ideality: The synthesis of (+)-kalkitoxin and (+)-hydroxyphthioceranic acid by assembly-line synthesis. J. Am. Chem. Soc. 137, 4398-4403 (2015). doi:10.1021/ja512875g Medline

38. H. Li, L. Wang, Y. Zhang, J. Wang, Transition-metal-free synthesis of pinacol alkylboronates from tosylhydrazones. Angew. Chem. Int. Ed. 51, 2943-2946 (2012). doi:10.1002/anie.201108139 Medline

39. V. Bagutski, A. Ros, V. K. Aggarwal, Improved method for the conversion of pinacolboronic esters into trifluoroborate salts: Facile synthesis of chiral secondary and tertiary trifluoroborates. Tetrahedron 65, 9956-9960 (2009). doi:10.1016/j.tet.2009.10.002

40. G. A. Molander, L. N. Cavalcanti, Oxidation of organotrifluoroborates via oxone. J. Org. Chem. 76, 623-630 (2011). doi:10.1021/jo102208d Medline

41. S. D. Dreher, P. G. Dormer, D. L. Sandrock, G. A. Molander, Efficient cross-coupling of secondary alkyltrifluoroborates with aryl chlorides-Reaction discovery using parallel microscale experimentation. J. Am. Chem. Soc. 130, 9257-9259 (2008). doi:10.1021/ja8031423 Medline

42. W. F. Bailey, X. Jiang, Cyclization of (4-methoxy-5-hexenyl)lithium. J. Org. Chem. 59, 6528-6533 (1994). doi:10.1021/j000101a010

43. R. Larouche-Gauthier, T. G. Elford, V. K. Aggarwal, Ate complexes of secondary boronic esters as chiral organometallic-type nucleophiles for asymmetric synthesis. J. Am. Chem. Soc. 133, 16794-16797 (2011). doi:10.1021/ja2077813 Medline

44. A. Bonet, M. Odachowski, D. Leonori, S. Essafi, V. K. Aggarwal, Enantiospecific $s p^{2}$ $s p^{3}$ coupling of secondary and tertiary boronic esters. Nat. Chem. 6, 584-589 (2014). doi:10.1038/nchem.1971 Medline

45. M. Burns, S. Essafi, J. R. Bame, S. P. Bull, M. P. Webster, S. Balieu, J. W. Dale, C. P. Butts, J. N. Harvey, V. K. Aggarwal, Assembly-line synthesis of organic molecules with tailored shapes. Nature 513, 183-188 (2014). doi:10.1038/nature13711 Medline

46. T. E. Barder, S. D. Walker, J. R. Martinelli, S. L. Buchwald, Catalysts for SuzukiMiyaura coupling processes: Scope and studies of the effect of ligand structure. J. Am. Chem. Soc. 127, 4685-4696 (2005). doi:10.1021/ja042491j Medline

47. N. Hartmann, M. Niemeyer, Easy large-scale synthesis of sterically encumbered 2 iodobiphenyls and some derivatives thereof. Syn. Comm. 31, 3839-3845 (2001). 
doi:10.1081/SCC-100108234

48. J. D. Hicks, A. M. Hyde, A. M. Cuezva, S. L. Buchwald, Pd-catalyzed N-arylation of secondary acyclic amides: Catalyst development, scope, and computational study. J. Am. Chem. Soc. 131, 16720-16734 (2009). doi:10.1021/ja9044357 Medline

49. N. C. Bruno, M. T. Tudge, S. L. Buchwald, Design and preparation of new palladium precatalysts for $\mathrm{C}-\mathrm{C}$ and $\mathrm{C}-\mathrm{N}$ cross-coupling reactions. Chem. Sci. 4, 916-920 (2013). doi:10.1039/C2SC20903A Medline

50. N. C. Bruno, N. Niljianskul, S. L. Buchwald, N-substituted 2aminobiphenylpalladium methanesulfonate precatalysts and their use in $\mathrm{C}-\mathrm{C}$ and C-N cross-couplings. J. Org. Chem. 79, 4161-4166 (2014). doi:10.1021/j0500355k Medline

51. S. D. Friis, T. Skrydstrup, S. L. Buchwald, Mild Pd-catalyzed aminocarbonylation of (hetero)aryl bromides with a palladacycle precatalyst. Org. Lett. 16, 4296-4299 (2014). doi:10.1021/ol502014b Medline

52. L. Li, C. Y. Wang, R. Huang, M. R. Biscoe, Stereoretentive Pd-catalysed Stille crosscoupling reactions of secondary alkyl azastannatranes and aryl halides. Nat. Chem. 5, 607-612 (2013). doi:10.1038/nchem.1652 Medline

53. S. A. Green, J. L. Matos, A. Yagi, R. A. Shenvi, Branch-selective hydroarylation: lodoarene-olefin cross-coupling. J. Am. Chem. Soc. 138, 12779-12782 (2016). doi:10.1021/jacs.6b08507 Medline

54. W. M. Czaplik, M. Mayer, A. J. V. Wangelin, Direct cobalt-catalyzed cross-coupling between aryl and alkyl halides. Synlett 18, 2931-2934 (2009).

55. A. Wilsily, Y. Nguyen, E. Fillion, Hydrogenolysis of unstrained carbon-carbon o bonds: Stereoselective entry into benzylic tertiary centers. J. Am. Chem. Soc. 131, 15606-15607 (2009). doi:10.1021/ja9076815 Medline

56. J. E. Baldwin, S. Bonacorsi Jr., Stereochemistry of the thermal isomerizations of $(1 R, \quad 2 R)-1-((E)$-styryl)-2-methylcyclopropane to 3-phenyl-4methylcyclopentenes. J. Am. Chem. Soc. 115, 10621-10627 (1993). doi:10.1021/ja00076a021

57. Schrödinger Release 2018-1: MacroModel (Schrödinger, LLC, New York, 2018).

58. K. Ermanis, K. E. B. Parkes, T. Agback, J. M. Goodman, Expanding DP4: Application to drug compounds and automation. Org. Biomol. Chem. 14, 3943-3949 (2016). doi:10.1039/C60B00015K Medline

59. K. Ermanis, J. M. Goodman, PyDP4 workflow integrating MacroModel/TINKER, Gaussian/NWChem and DP4 analysis, version 0.7 (2016); https://github.com/KristapsE/PyDP4/.

60. M. J. Frisch, G. W. Trucks, H. B. Schlegel, G. E. Scuseria, M. A. Robb, J. R. Cheeseman, G. Scalmani, V. Barone, G. A. Petersson, H. Nakatsuji, X. Li, M. Caricato, A. V. Marenich, J. Bloino, B. G. Janesko, R. Gomperts, B. Mennucci, H. P. Hratchian, J. V. Ortiz, A. F. Izmaylov, J. L. Sonnenberg, D. Williams-Young, F. Ding, F. Lipparini, F. Egidi, J. Goings, B. Peng, A. Petrone, T. Henderson, D. Ranasinghe, V. G. Zakrzewski, J. Gao, N. Rega, G. Zheng, W. Liang, M. Hada, M. Ehara, K. Toyota, R. Fukuda, J. Hasegawa, M. Ishida, T. Nakajima, Y. Honda, O. Kitao, H. Nakai, T. Vreven, K. Throssell, J. A. Montgomery Jr., J. E. Peralta, F. Ogliaro, M. J. Bearpark, J. J. Heyd, E. N. Brothers, K. N. Kudin, V. N. Staroverov, T. A. Keith, R. Kobayashi, J. Normand, K. Raghavachari, A. P. Rendell, J. C. Burant, S. S. Iyengar, J. Tomasi, M. Cossi, J. M. Millam, M. Klene, C. Adamo, R. Cammi, J. W. Ochterski, R. L. Martin, K. Morokuma, O. Farkas, J. B. Foresman, D. J. Fox, Gaussian 16, Revision A.03 (Gaussian, Inc., Wallingford, CT, 2016).

61. C. Adamo, V. Barone, Toward reliable density functional methods without adjustable parameters: The PBE0 model. J. Chem. Phys. 110, 6158-6170 (1999). doi:10.1063/1.478522

62. W. J. Hehre, R. Ditchfield, J. A. Pople, Self-consistent molecular orbital methods. XII. Further extensions of Gaussian-type basis sets for use in molecular orbital studies of organic molecules. J. Chem. Phys. 56, 2257-2261 (1972). doi:10.1063/1.1677527

63. P. C. Hariharan, J. A. Pople, The influence of polarization functions on molecular orbital hydrogenation energies. Theor. Chim. Acta 28, 213-222 (1973). doi:10.1007/BF00533485

64. T. Clark, J. Chandrasekhar, G. W. Spitznagel, P. V. R. Schleyer, Efficient diffuse function-augmented basis sets for anion calculations. III. The $3-21+G$ basis set for first-row elements, Li-F. J. Comput. Chem. 4, 294-301 (1983). doi: $10.1002 /$ jcc. 540040303

65. R. Krishnan, J. S. Binkley, R. Seeger, J. A. Pople, Self-consistent molecular orbital methods. XX. A basis set for correlated wave functions. J. Chem. Phys. 72, 650654 (1980). doi:10.1063/1.438955

66. J. R. Cheeseman, G. W. Trucks, T. A. Keith, M. J. Frisch, A comparison of models for calculating nuclear magnetic resonance shielding tensors. J. Chem. Phys. 104, 5497-5509 (1996). doi:10.1063/1.471789

67. Y. Zhao, D. G. Truhlar, The M06 suite of density functionals for main group thermochemistry, thermochemical kinetics, noncovalent interactions, excited states, and transition elements: Two new functionals and systematic testing of four M06-class functionals and 12 other function. Theor. Chem. Acc. 120, 215-241 (2008). doi:10.1007/s00214-007-0310-x

68. F. Weigend, R. Ahlrichs, Balanced basis sets of split valence, triple zeta valence and quadruple zeta valence quality for $\mathrm{H}$ to Rn: Design and assessment of accuracy. Phys. Chem. Chem. Phys. 7, 3297-3305 (2005). doi:10.1039/b508541a Medline

69. E. D. Glendening, A. E. Reed, J. E. Carpenter, F. Weinhold, NBO Version 3.1 (University of Wisconsin-Madison, 1990).

70. S. K. Latypov, F. M. Polyancev, D. G. Yakhvarov, O. G. Sinyashin, Quantum chemical calculations of ${ }^{31} \mathrm{P}$ NMR chemical shifts: Scopes and limitations. Phys. Chem. Chem. Phys. 17, 6976-6987 (2015). doi:10.1039/C5CP00240K Medline

71. H. Clavier, S. P. Nolan, Percent buried volume for phosphine and $N$-heterocyclic carbene ligands: Steric properties in organometallic chemistry. Chem. Commun. (Camb.) 46, 841-861 (2010). doi:10.1039/b922984a Medline

72. L. Falivene, R. Credendino, A. Poater, A. Petta, L. Serra, R. Oliva, V. Scarano, L. Cavallo, SambVca 2. A web tool for analyzing catalytic pockets with topographic steric maps. Organometallics 35, 2286-2293 (2016) doi:10.1021/acs.organomet.6b00371

73. SambVca 2.0 server; www.molnac.unisa.it/OMtools/sambvca2.0/index.html.

74. A. Verloop, in Drug Design, E. J. Ariens, Ed. (Academic Press, 1976), vol. 3, pp. 133 187

75. T. Piou, F. Romanov-Michailidis, M. Romanova-Michaelides, K. E. Jackson, N. Semakul, T. D. Taggart, B. S. Newell, C. D. Rithner, R. S. Paton, T. Rovis, correlating reactivity and selectivity to cyclopentadienyl ligand properties in Rh(III)-catalyzed C-H activation reactions: An experimental and computational study. J. Am. Chem. Soc. 139, 1296-1310 (2017). doi:10.1021/jacs.6b11670 Medline

76. K. Jackson, R. Paton, Sterimol.py (2017) https://github.com/bobbypaton/Sterimol.

77. I. A. Guzei, M. Wendt, An improved method for the computation of ligand steric effects based on solid angles. Dalton Trans. 2006, 3991-3999 (2006). doi:10.1039/b605102b Medline

78. I. A. Guzei, M. Wendt, Program Solid-G (University of Wisconsin-Madison, 2004). 79. MATLAB R2017a (Mathworks, Inc., Natick, MA, 2017).

80. J.-Y. Guo, Y. Minko, C. B. Santiago, M. S. Sigman, Developing comprehensive computational parameter sets to describe the performance of pyridine-oxazoline and related ligands. ACS Catal. 7, 4144-4151 (2017). doi:10.1021/acscatal.7b00739

\section{ACKNOWLEDGMENTS}

We acknowledge R. Kinthada for contributions to the diastereoselectivity studies. We thank G. Ralph for assistance with chiral HPLC analysis. We thank Prof. Luigi Cavallo for providing us with the command line tool for computing percent buried volume. Funding: we are grateful to the National Institutes of Health (grant SC1GM110010 to M.R.B.), the National Science Foundation (grant CHE1665189 to M.R.B. and grants CHE-1361296 and CHE-1763436 to M.S.S), and the Leopoldina Fellowship Programme of the German National Academy of Sciences Leopoldina (LPDS 2017-18 to T.G.). The support and resources from the Center for High Performance Computing (CHPC) at the University of Utah are gratefully acknowledged. Further computational resources were provided by the Extreme Science and Engineering Discovery Environment (XSEDE), which is supported by the NSF (ACI-1548562) and provided through allocation TG-CHE180003. Author contributions: S.Z. and B.M. performed all synthetic experiments and isolated all products. T.G. computed all ligand parameters and performed multivariate analyses. Z.L.N. performed initial multivariate analyses. M.R.B., M.S.S., and T.G. wrote the manuscript. M.R.B. and M.S.S. directed the project. Competing interests: The authors declare no competing interests. Data and materials availability: All additional data are in the supplementary materials. 


\section{SUPPLEMENTARY MATERIALS}

www.sciencemag.org/cgi/content/full/science.aat2299/DC1

Materials and Methods

Supplementary Text

Fig. S1

Tables S1 to S6

NMR Spectra

References (38-80)

18 May 2018; accepted 6 September 2018

Published online 20 September 2018

10.1126/science.aat2299 


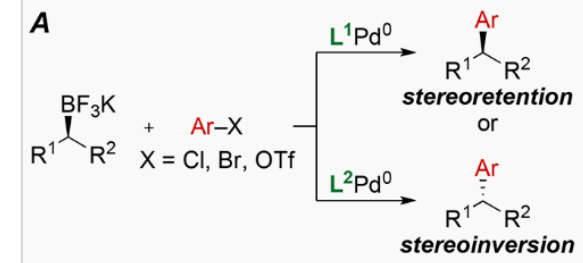

Using reaction discovery guided by modern statistical data analysis, we have achieved:

- rapid and predictable three-dimensional structural diverisification

- ligand-determined stereocontrol of products - a predictive mechanistic model for alkylboron transmetallation
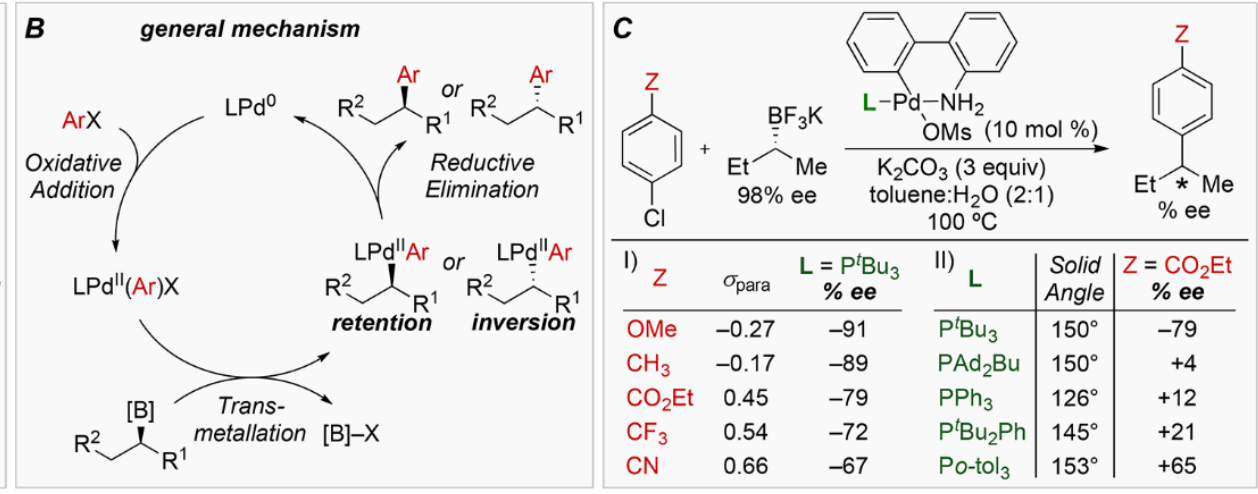

Fig. 1. Reaction development. (A) Enantiodivergent Suzuki reactions of secondary alkylboron nucleophiles. (B) General mechanism. (C) Initial investigation of substrate and ligand influences on stereoselectivity. $+\%$ ee $=$ net retention, $-\%$ ee $=$ net inversion. 


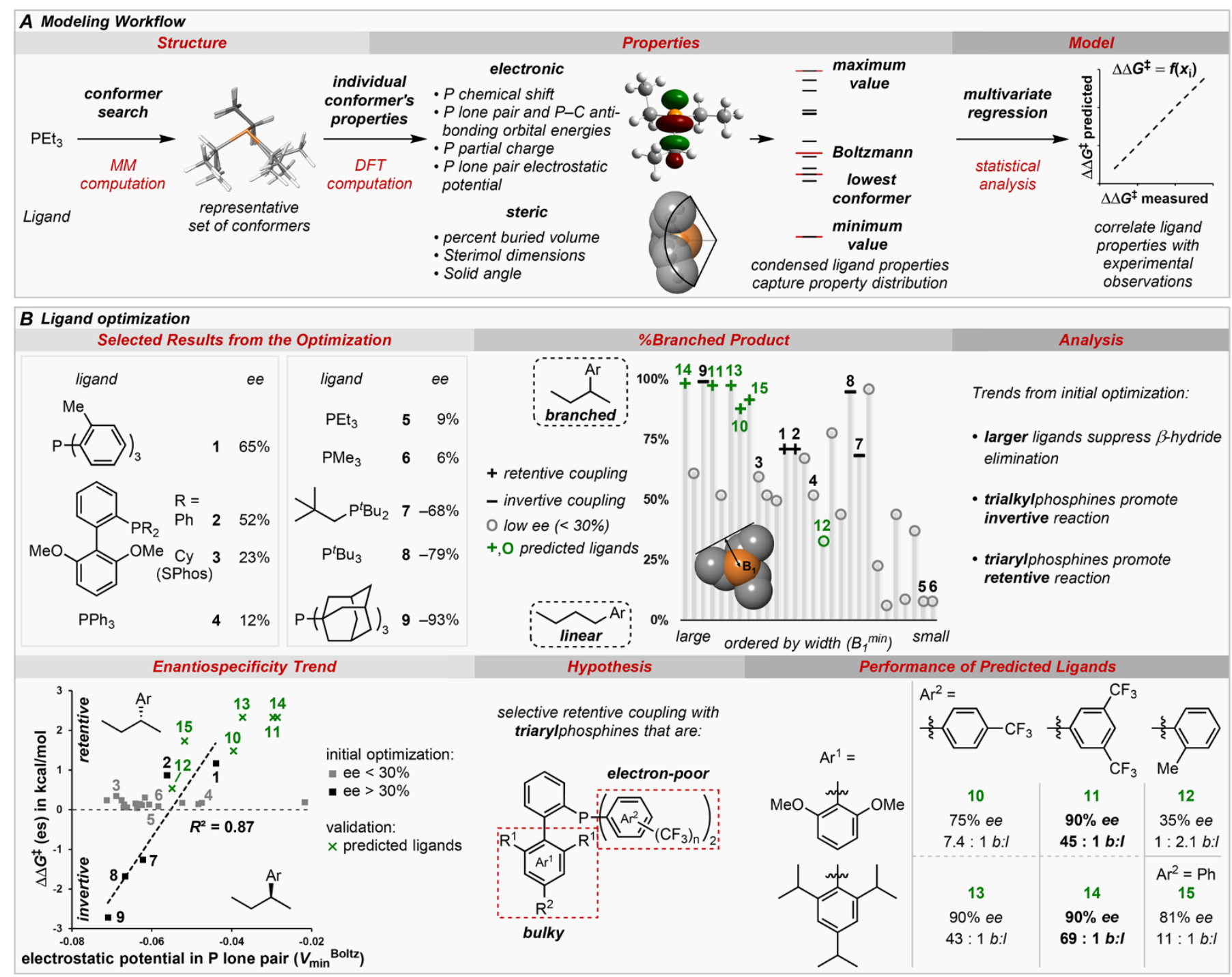

Fig. 2. Phosphine parameterization. (A) Workflow of parameter generation and statistical modeling. (B) Application of phosphine parameterization to ligand optimization of the reaction shown in Fig. $1 \mathrm{C}$ with $\mathrm{Z}=\mathrm{CO}_{2} \mathrm{Et}$. b:I = branched to linear ratio. $+\%$ ee $=$ net retention, $-\%$ ee $=$ net inversion. 

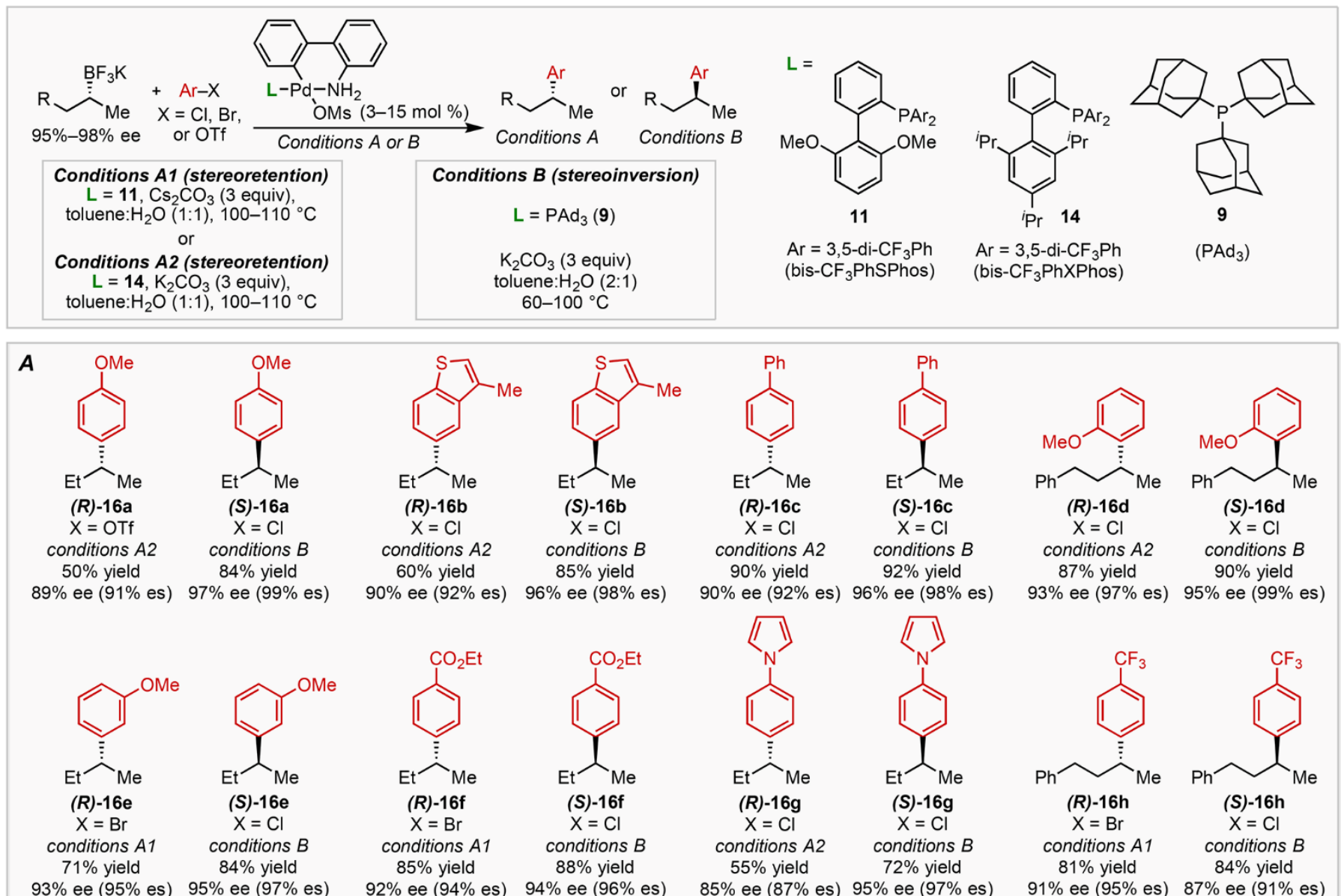
$93 \%$ ee $(95 \%$ es $) \quad 95 \%$ ee $(97 \%$ es $)$ $\begin{array}{cc}85 \% \text { yield } & 88 \% \text { yield } \\ 92 \% \text { ee }(94 \% \text { es }) & 94 \% \text { ee }(96 \% \text { es })\end{array}$ $85 \%$ ee ( $(87 \%$ es) $95 \%$ ee ( $97 \%$ es)
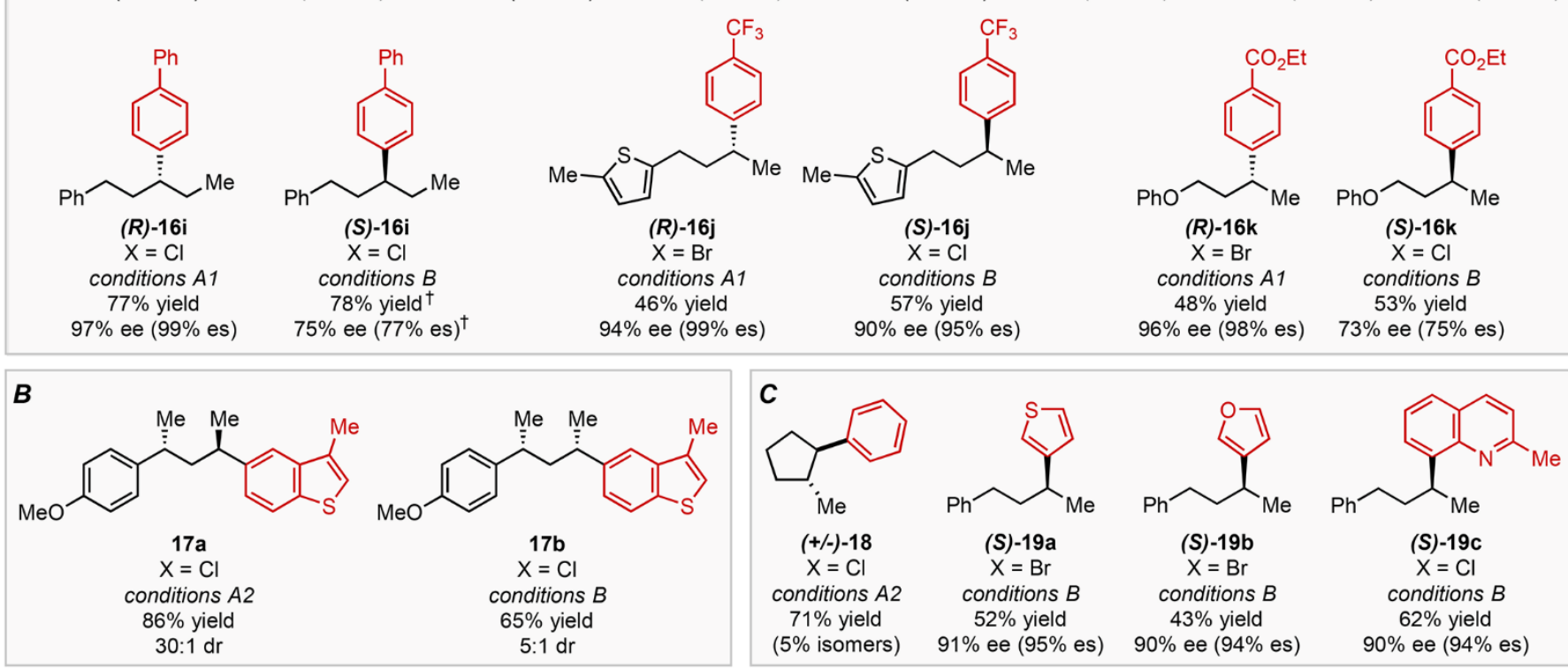

Fig. 3. Stereodivergent Pd-catalyzed cross-coupling reactions using enantioenriched alkylboron nucleophiles. The general reaction scheme is shown at the top. (A to C) Isolated yields are shown for stereoretentive and stereoinvertive cross-coupling reactions (A), diastereoselective cross-coupling reactions (B), and additional individual reactions (C). $\%$ es $=\%$ ee (final product) $/ \%$ ee (starting material). $\uparrow 44 \%$ yield, $84 \%$ ee ( $86 \%$ es) when run at $60^{\circ} \mathrm{C}$. 


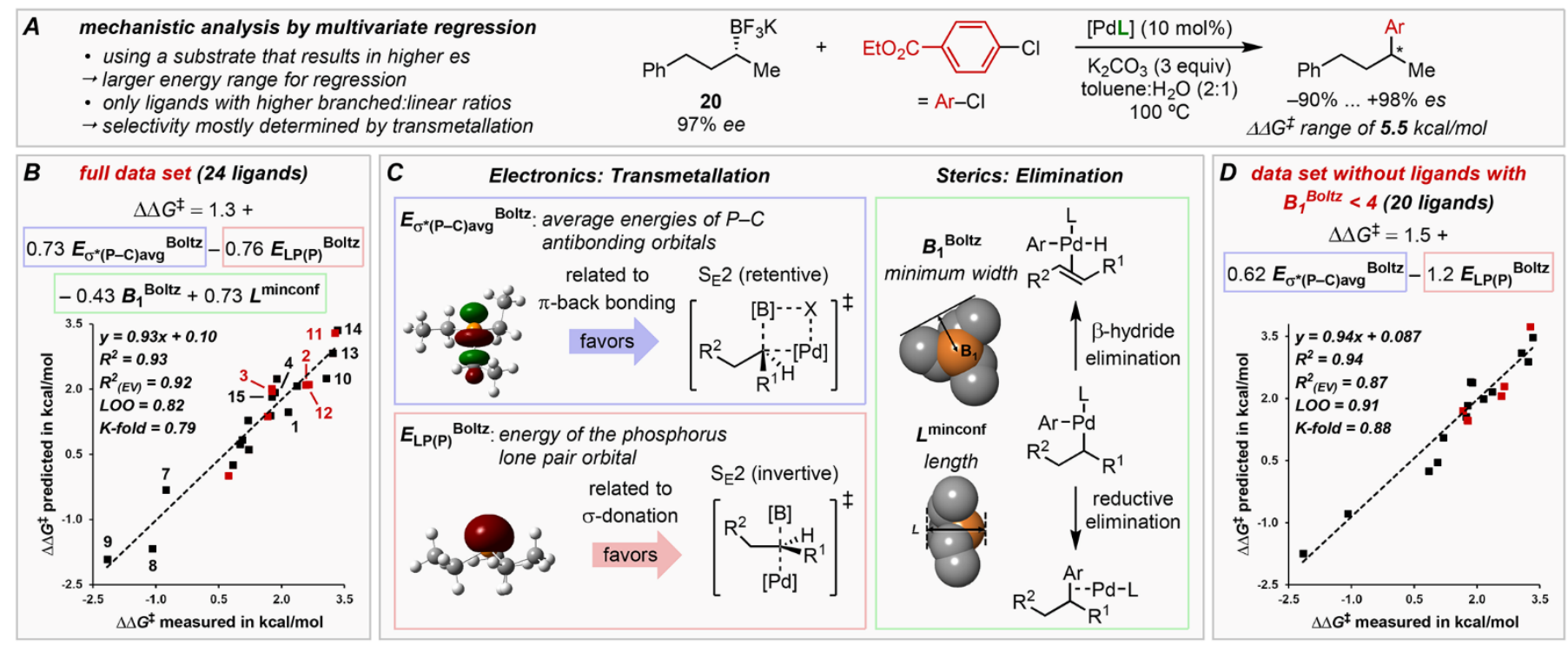

Fig. 4. Mechanistic investigation by multidimensional regression modeling. (A) Data from the arylation of 20 was used. $[\mathrm{PdL}]$ is the precatalyst as shown in Fig. 3 with varying ligands. (B) Regression model containing all 24 ligands of this data set. $E_{\sigma^{*}(\mathrm{P}-\mathrm{C}) \text { avg }}{ }^{\text {Boltz: }}$ Boltzmann-weighted average across the conformers of the average energies of the three $\mathrm{P}-\mathrm{C} \sigma^{*}$ antibonding orbitals in each phosphine. $\left.E_{\mathrm{LP}(\mathrm{P})}\right)^{\text {Boltz: }}$ : Boltzmann-weighted average of the energy of the phosphorus lone pair orbital. Sterimol $B_{1}{ }^{B o l t z}$ is the least width and Sterimol $L$ minconf is the length of the lowest-energy conformer as seen from opposite the $P$ substituents. Red points in the diagram: validation data (EV) not used in the model training. $\mathrm{LOO}=$ leave-one-out cross validation score. $\mathrm{K}$-fold: average threefold cross-validation score. (C) Illustration and interpretation of the model terms. (D) Regression model after removing the four smallest ligands in this data set to exclude the influence of competitive $\beta$-hydride elimination on the data. 